\title{
Histochemical and ultrastructural studies of the mosquito Aedes aegypti fat body: effects of aging and diet type
}

\author{
Gustavo Ferreira Martins ${ }^{1,}$, , José Eduardo Serrão1 ${ }^{1}$, José Marcelo Ramalho-Ortigão², and \\ Paulo Filemon Paolucci Pimenta ${ }^{3}$ \\ ${ }^{1}$ Departamento de Biologia Geral, Universidade Federal de Viçosa, Viçosa, MG, Brazil, \\ 36570-000 \\ 2 Department of Entomology, Kansas State University, Manhattan, KS, 66506, USA \\ ${ }^{3}$ Laboratório de Entomologia Médica, Instituto René Rachou, Fundação Oswaldo Cruz, Belo \\ Horizonte, MG, Brazil, 30190-002
}

\begin{abstract}
Aedes aegypti is the principal vector of dengue world wide and a major vector of urban yellow fever. Despite its epidemiological importance, not much is known regarding cellular and structural changes in the fat body in this mosquito. Here, we applied light and transmission electron microscopies in order to investigate structural changes in the fat body of three groups of A. aegypti females: newly emerged, 18-day-old sugar-fed, and 18-day-old blood-fed. The fat body consists of a layer of cells attached to the abdomen integument, formed by trophocytes and oenocytes. Trophocytes are strongly positive for carbohydrates, while oenocytes are strongly positive for proteins and lipids. Ultrastructural analyses of trophocytes from newly emerged and 18-day-old blood-fed indicate that these cells are rich in glycogen and free ribosomes. Many lipid droplets and protein granules, which are broken down after the blood meal, are also detected. In 18-day-old sugar-fed, trophocytes display a disorganized cytoplasm filled with lipid droplets, and reduced numbers of free ribosomes, glycogen, rough endoplasmic reticulum (RER) and mitochondria. Following a blood meal, the RER and mitochondria display enlarged sizes, suggestive of increased activity. In regards to oenocytes, these cells display an electron-dense cytoplasm and plasma membrane infoldings facing the hemolymph. As the A. aegypti female ages, trophocyte and oenocyte cell nuclei become larger but decrease in diameter after blood feeding. Our findings suggest that the trophocytes and oenocytes remodeling is likely involved in functional changes of fat body that take place during aging and following a blood meal in A. aegypti females.
\end{abstract}

\section{Keywords}

Aedes aegypti; trophocytes; oenocytes; morphology; microscopy; fat body; mosquito; histochemistry

\section{Introduction}

The fat body is the major metabolic organ of insects. It is a diffuse organ, occurring as sheets or lobes of tissue lining the body cavity, primarily the abdomen (Chapman, 1998), and is involved in the storage and transport of many precursors that are eventually released

\footnotetext{
"Corresponding author: Gustavo F Martins, Departamento de Biologia Geral, Universidade Federal de Viçosa, Viçosa, Minas Gerais, Brazil, CEP 36570-000; Fax\# 5531 3891-2549; gmartins@ufv.br.
} 
into the hemolymph (Tucker, 1977; Haunerland and Shirk, 1995; Paskewitz and Christensen, 1996, Gillespie et al., 1997; Tzou et al., 2002; Hetru et al., 2003).

According to the insect order, the fat body may contain a variety of cell types such as trophocytes, urate cells (for uric acid storage), mycetocytes (containing symbiotic bacteria), and oenocytes. However, in Diptera (including mosquitoes), only trophocytes and oenocytes are present (Wigglesworth 1942; Sohal, 1973; Stoppie et al., 1981; Johnson \& Batterworth, 1985). Trophocytes are the major cell type and constitute the basic structure of the organ. They have a cytoplasm that is rich in lipid droplets, protein granules, sugars and yolk precursors, free ribosomes, rough endoplasmic reticulum, and Golgi complex (Behan and Hagedorn, 1978; Raikhel and Lea, 1983; Raikhel, 1986a, b; Grzelak and Kumaran, 1986; Wang and Haunerland, 1991; 1992; Easton and Horwath, 1994; Snigirevskaya et al., 1997).

The morphology of fat body cells changes during insect post-embryonic development, and also depends on the insect nutrition and on the period of its reproductive stage (Haunerland and Shirk, 1995; Chapman, 1998; Martins and Pimenta, 2008). In blood feeding insects such as Aedes aegypti, the morphology of such cells varies during the gonotrophic cycle; and depending on the time after blood feeding the modifications may include replication and turnover of organelles, and uptake and depletion of stored substances (Raikhel \& Lea, 1983; Raikhel, 1986a, b; Snigirevskaya et al., 1997).

The aim of this study was to characterize the morphology, histochemistry, and subcellular aspects of $A$. aegypti fat body by comparing female mosquitoes at different age and diet (sugar vs. blood). Based on various criteria including cell inclusions, nuclei size, and organelles profile our findings demonstrate that mosquito fat body undergoes significant changes associated with aging and blood feeding.

\section{Materials and Methods}

\section{Mosquitoes}

Females A. aegypti (strain PP-Campos, Campos dos Goytacazes, RJ, Brazil) were obtained from a colony maintained at the Laboratory of Medical Entomology of the Instituto René Rachou (IRR-FIOCRUZ, MG, Brazil). Three groups of females were used: (1) newly emerged; (2) 18-day-old non blood-fed or sugar-fed; and (3) 18-day-old blood-fed on anesthetized mice 4 days before the time of the dissection. All mosquito groups received sugar solution ad libitum until the time of dissection.

\section{Dissection and sample preparation}

Mosquitoes were dissected under a stereoscope microscope in $300 \mu \mathrm{L}$ sterile PBS (Phosphate Buffer Saline). The abdomen was separated from the thorax and opened longitudinally to expose the fat body attached to the integument. Twenty-five $\mu$ l of a fixative solution containing $2.5 \%$ glutaraldehyde in PBS supplemented with $7 \%$ sucrose was gently added directly onto the fat body adjacent to the integument. After dissection, this abdominal fat body tissue was maintained in the fixative solution until further use in the experiments described below.

\section{Transmission electron microscopy (TEM)}

Abdominal fragments were fixed in $2.5 \%$ glutaraldehyde in $0.1 \mathrm{M}$ cacodylate buffer $\mathrm{pH} 7.2$ and post-fixed in $1 \%$ osmium tetroxide plus $0.8 \%$ potassium ferricyanide in the same buffer (Pimenta and De Souza, 1983). Samples were dehydrated in a crescent series of 30-100\% acetone and embedded in Epon-Araldite resin (Electron Microscopy Sciences, Hatfield, PA). Semi-thin sections were stained with $1 \%$ toluidine blue-borax to be observed in the light 
microscope. The ultra-thin sections were stained with uranyl acetate and lead citrate (Reynolds, 1963). After the staining, the ultra-thin sections were washed in distilled water and analyzed under a transmission electron microscope (TEM Zeiss model EM 109).

\section{Histology and histochemistry}

Additional samples fixed as described above were rinsed in sterile PBS, dehydrated in crescent series of 70-100\% ethanol, and embedded in Historesin (Leica). Whole fixed mosquitoes without heads and appendices also were processed for histological in situ examination of the fat body. Two- $\mu \mathrm{m}$ sections were obtained from whole mosquito samples and stained with $1 \%$ toluidine blue-borax.

For histochemical analyses, four separate staining methods were applied: (a) Mercurybromophenol blue for protein detection; (b) Periodic acid-Schiff (PAS) for polysaccharide and glycoconjugates; (c) Feulgen reaction for DNA counterstained by fast green $0.1 \%$; and (d) $2 \%$ osmium tetroxide in cacodylate buffer $\mathrm{pH} 7.4$ for lipid detection. All methods were adapted from Behmer and collaborators (1976).

\section{Morphometric and statistical analyzes}

Nuclei measurements of trophocytes and oenocytes were taken from histological sections using the software Zeiss KS $300^{\mathrm{TM}}$. A total of two experimental groups were analyzed with six specimens per group. The values obtained were compared by variance analysis (ANOVA) and Tukey test, and the level of significance was set at 5\%

\section{Results}

\section{Histology and histochemistry}

In newly emerged $A$. aegypti females the fat body is formed by a cell mass underneath the integument with varying thickness along the body cavity (Figure 1a). Similar observations were made using 18-day-old sugar-fed or blood-fed females. Additionally, the fat body can be seen either in direct contact with Malpighian tubules and the digestive tract, or separated from these and other organs by muscle layers, as shown in Figures 1a-c. Muscular and tracheal elements can also be observed.

Two cell types are distinctly recognized: trophocytes and oenocytes (Figures 1c-d). Trophocytes are morphologically uniform displaying a globular shape, a pleomorphic nucleus, and the cytoplasm squeezed among many lipid inclusions. Oenocytes have a central and large nucleus, and a more homogeneous and basophilic cytoplasm in comparison to trophocytes. Although oenocytes can be found either in close proximity to each other or isolated by the trophocytes, they are preferentially located in the periphery of the organ (Figure 1c). A well-developed nucleolus can be seen in both trophocytes and oenocytes. Cell elongations are sometimes also present in oenocytes (Figure 1d). Additionally, in adult females, the percentages of oenocytes and trophocytes vary from 5.9 to $9.8 \%$ and from 94.1 to $91.2 \%$, respectively.

Trophocytes and oenocytes were readily distinguishable by the staining procedures used. Additionally, the cytoplasm in trophocytes of newly emerged mosquitoes displayed strongly positive staining for the PAS test in contrast to the weak staining shown by 18-day-old fed females (data not shown). For oenocytes, cytoplasms were consistently negative for the PAS staining regardless of mosquito age or feeding condition (Figure 1e).

Bromophenol blue staining revealed that in newly emerged females the trophocyte cytoplasm is positive for proteins, with the protein granules being abundant and strongly 
stained in the periphery of the cell (Figure 2a). Protein granules are fewer and smaller in trophocytes from 18-day-old blood-fed than in newly emerged females, and are homogenously stained by bromophenol blue (Figure $2 b$ ). Again, in contrast to trophocytes, the oenocytes were strongly and uniformly stained for proteins (Figure 2a). Regarding lipid content, the osmium tetroxide staining indicated that trophocytes have a cytoplasm rich in lipid droplets. For newly emerged females, lipid droplets detected within trophocytes are spherical and smaller than in either sugar or blood-fed females. In oenocytes, lipid droplets were not detected within the cytoplasm, despite the homogeneous stain detected by osmium tetroxide (Figures 2c-d).

\section{Ultrastructure}

The TEM analyses revealed that in newly emerged females, trophocytes display a cytoplasm filled with lipid droplets and glycogen. The nucleus displayed variable morphology, with an evident nucleolus and regions of condensed chromatin. Electron-dense granules also were present, with the cytoplasm rich in clusters of free ribosomes. Mitochondria were detected mainly around the nucleus, close to lipid droplets and beneath the cytoplasmic membrane. The rough endoplasmic reticulum (RER) and Golgi complex are well-developed (Figures $3 \mathrm{a}-\mathrm{c})$. Regarding oenocytes, the plasma membrane displays several infoldings while a large number of mitochondria were detected within the cytoplasm. Mitochondria and the RER are located near the nucleus, and a condensed chromatin is visible on the nuclear periphery (Figures $3 \mathrm{~d}-\mathrm{e}$ ). In addition, parts of the oenocyte cytoplasm displayed a well developed SER (Figure 4f).

In 18-day-old sugar-fed females, the trophocyte cytoplasm is almost completely filled by lipid droplets. The RER and Golgi are only slightly developed, and glycogen and free ribosomes are scarce. Electron-dense structures are seen in the cytoplasm and the mitochondrial profiles display reduced crystae. The nucleus is well developed with a large nucleolus and squeezed between lipid droplets (Figs. 3f, 4a-b).

In 18-day-old blood-fed females, trophocytes displayed a cytoplasm rich in glycogen. The lipid droplets of the blood-fed are smaller than in the trophocytes the sugar-fed and more developed than in trophocytes of newly emerged. The cytoplasm of 18-day-old blood-fed is also rich in mitochondria with a dilated profile and enlarged crystae. Lisosome-like electrondense bodies filled by fibrous material are common in the trophocyte cytoplasm of these females (Figs. 4c-d).

In both sugar and blood-fed females, the infoldings detected in the plasma membrane of oenocytes are more developed than in newly emerged females. There are also more numerous infoldings facing the hemolymph (Fig. 4e).

\section{Morphometric analyses}

The results obtained from measurements of nuclei diameter for the different $A$. aegypti female groups analyzed are summarized in Table 1. For trophocytes, the diameter of the nucleus becomes larger with age, and is smaller in newly emerged females in comparison with the two others two treatments (i.e., sugar and blood fed) $(\mathrm{p}<0.001)$. Overall, the largest trophocyte nuclear diameter is that of 18-day-old sugar-fed females $(\mathrm{p}<0.01)$.

For oenocytes, diameter sizes were not statistically different between newly emerged and 18-day-old sugar-fed females ( $p>0.05$ ). However, the nuclear diameters were significantly different $(p<0.001)$ between these two groups in comparison with that of 18-day-old blood fed females, whose nuclear diameter were consistently smaller. 


\section{Discussion}

The cellular organization of $A$. aegypti adult females fat body is similar to that of larval stages (Wigglesworth, 1942), and to what was described in other Diptera (Sohal, 1973; Tobe et al., 1973; Stoppie et al., 1981; Dean et al., 1985; Johnson and Butterworth et al., 1988).

Trophocytes constitute the major cell type. They are globular cells with the cytoplasm filled with lipids, sugars and proteins (Behan \& Hagedorn, 1978; Raikhel and Lea, 1983; Raikhel 1986a, b; Snigirevskaya et al., 1997). Adult A. aegypti trophocytes display a strong positive reaction for the PAS test, likely associated with their role in sugar storage in insects (Chapman, 1998). In contrast, oenocytes which are significantly less represented in the $A$. aegypti fat body display a centralized nucleus and a dense and homogeneous cytoplasm. Additionally, oenocytes have fewer lipid and glycogen granules than trophocytes (Sohal, 1973; Tobe et al., 1973; Stoppie et al., 1981; Dean et al., 1985).

Cytoplasm from trophocytes dissected from 18-day-old sugar- and blood-fed females have increased lipid storage droplets compared to newly emerged females, suggesting that lipids are synthesized and stored in the insect fat body during periods of active feeding (Raikhel and Lea, 1983). Protein granules on the other hand are abundant in newly emerged, and become scarce in 18-day-old sugar and blood-fed A. aegypti females. This decrease in proteins granules is probably associated with the lack of protein content in sugar fed mosquitoes, and with the need to supply yolk proteins (produced by trophocytes) for ovarian development in blood fed females.

Although of ectodermal origin, the anatomical location of oenocytes varies considerably amongst insect species and even across different developmental stages. In the kissing bug Rhodnius prolixus and in the cockroach Blattella germanica, oenocytes are located close to their point of origin in the epidermis (Wigglesworth, 1988; Fan et al., 2003). In Calpodes ethlius (Lepidoptera), these cells are situated close to specialized wax glands (Larsen, 1976). For adult $A$. aegypti females, our results indicate that oenocytes are located preferentially in the periphery of the fat body, organized in cell clusters or dispersed between trophocytes.

Histochemically, A. aegypti female oenocytes displayed a PAS-negative cytoplasm which also was strongly and uniformly stained by bromophenol blue and osmium. These results are suggestive of protein and lipid synthesis, as observed in oenocytes of other insects (Fan et al., 2003; Gutierrez et al., 2007).

We noticed that trophocytes of newly emerged A. aegypti are similar to trophocytes studied in the previtellogenic stage of other Diptera. In general, these cells are characterized by an abundance of lipid droplets, glycogen, RER, Golgi complex, mitochondria, and large spherical and dense protein granules (Thomsen \& Thomsen, 1974; Tobe and Davey, 1974; Stoppie et al., 1981). However, in trophocytes from 18-day-old blood- or sugar-fed $A$. aegypti, the number of such organelles is reduced.

Trophocytes are the principal storage cell for lipids in insects. Some of the most prominent changes detected in trophocytes of 18-day-old sugar-fed mosquitoes were an increase in the size of lipid droplets, and reduced glycogen content. Our results corroborate the finding that lipid amounts increase in the fat body during A. aegypti aging (Ziegler and Ibrahin, 2001). The lipid droplets in trophocytes of 18-day-old sugar-fed were larger, filling almost all the cytoplasm, than those of 18-day-old blood-fed. Suggestive of the ability of the mosquito fat body to store lipid from sugar supplies during aging. Thus, confirming that sugar feeding in A. aegypti is necessary to maintain the energy reserves preceding the mosquito reproduction. Also, the increased size of lipid droplets in the trophocyte cytoplasm following blood 
feeding might be a result of lipid synthesis prior to yolk supplementation (Ziegler and Ibrahin, 2001).

Large amounts of vitellogenic proteins must be produced during A. aegypti vitellogenesis. As cells specialized in protein synthesis, trophocytes display an abundance of RER, Golgi complex, and secretory granules until approximately $24 \mathrm{~h}$ after blood-meal. However, $36-$ to- $42 \mathrm{~h}$ after the blood meal, the protein synthesis apparatus rapidly degenerate. The scarcity of RER and Golgi complex in the trophocytes of blood-fed females may be due to organelle turnover that probably extends until four days after blood meal as a consequence of lysosomal activity (Raikhel and Lea, 1983; Snigirevskaya et al., 1997). Our result demonstrating the presence of electron-dense elements resembling lysosomes also supports that notion.

Similar to our findings in A. aegypti, the major characteristic of oenocytes in insects is the presence of an extensive SER, of invaginations of the plasma membrane, and of a highly electron dense cytoplasm with Golgi complex and secretory granules scarcely developed (Sohal, 1973; Stoppie et al., 1981; Wigglesworth, 1988; Fan et al., 2003). In accordance to previous data (Locke, 1969; Tobe and Davey, 1974; Jackson and Locke, 1989; Fan et al., 2003), our findings suggest that oenocytes are involved in lipid metabolism. Also, the number of cell membrane invaginations, and the peripheral location observed for the oenocytes after blood feeding (or aging) suggest an increased oenocyte-hemolymph exchange in comparison to newly emerged $A$. aegypti females.

We observed an increase in the diameter of the cell nucleus during the development of the adult female fat body, as reported during the female gonotrophic cycle (Raikhel and Lea 1983). It has been shown that DNA amplification is responsible for trophocyte nuclei development of fruit fly larvae (Butterworth and Rasch, 1986; Butterworth et al., 1988), and in A. aegypti adult females three days after blood-feeding (Dittmanm et al., 1989). Likewise, the increased diameter observed for A. aegypti adult female nuclei may also be due to DNA amplification.

Our results indicate that nuclei in trophocytes vary in size according to diet, being larger in 18-day-old sugar- than blood-fed A. aegypti females. As protein synthesis in trophocytes can be correlated with nuclei development (Dittmanm et al., 1989), the decrease in nuclear diameter following blood feeding may be linked with the end of the vitellogenic process and the reduced activity of these cells four days after blood feeding (Snigirevskaya, et al., 1997; Raikhel, 1986b; Raikhel \& Lea, 1983). In the 18-day-old sugar-fed females, the larger diameter of the nucleus suggests an increase in cell activity during the lipogenesis process compared to newly emerged females (Ziegler and Ibrahin, 2001).

Oenocytes also demonstrated a difference in nuclear diameter in regards to diet. In 18-dayold sugar- and blood-fed females, the nuclei were enlarged in comparison to newly emerged. However, nuclei from sugar-fed females were larger than the ones in blood-fed females. Again, considering that oenocyte protein synthesis activity can be correlated with nuclei development, the differences detected for nuclear size may reflect an increase in protein synthesis.

This study reveals for the first time the morphological and functional modifications displayed by trophocytes and oenocytes from A. aegypti. The characterization of such cellular restructuring in fat body cells will assist with providing a roadmap to the full understanding of these cells in the metabolism of this major disease vector. 


\section{Acknowledgments}

This work was financially supported by Conselho Nacional de Desenvolvimento Científico e Tecnológico (CNPq), Programa Nacional de Excelência - PRONEX, Fundação de Amparo a Pesquisa de Minas Gerais (Fapemig) and Fundação Oswaldo Cruz (FIOCRUZ). JMR-O is funded by NIH grants AI074691 and AI083831. We also acknowledge the Núcleo de Microscopia e Microanálise, Universidade Federal de Viçosa, Minas Gerais, for technical assistance.

\section{References}

Behan M, Hagedorn HH. Ultrastructural changes in the fat body of adult female Aedes aegypti in relationship to vitellogenin synthesis. Cell Tissue Res. 1978; 186(3):499-506. [PubMed: 627029]

Behmer, OA.; Tolosa, EMC.; Freitas Neto, AG. São Paulo: EDART - São Paulo Livraria Editora. Editora da Universidade de São Paulo; 1976. Manual de técnicas para histologia normal e patológica; p. 241

Butterworth FM, Emerson L, Rasch EM. Maturation and degeneration of the fat body in the Drosophila larva and pupa as revealed by morphometric analysis. Tissue Cell. 1988; 20(2):255268. [PubMed: 3136556]

Butterworth FM, Rasch EM. Adipose tissue of Drosophila melanogaster: VII. Distribution of nuclear DNA amounts along the anterior-posterior axis in the larval fat body. J Exp Zool. 1986; 158:77-85. [PubMed: 2427640]

Chapman, RF. Structure and function. Cambridge: Cambridge University Press; 1998. The insects; p. 770

Dean, RL.; Locke, M.; Collins, JV. Structure of fat body. In: Kerkut, GA.; Gilbert, LI., editors. Comprehensive Insect Physiology, Biochemistry, and Pharmacology. Oxford: Pergamon; 1985. p. $155-210$.

Easton CM, Horwath KL. Characterization of primary cell cultures derived from fat body of the beetle, Tenebrio molitor, and the immunolocalization of a thermal hysteresis protein in vitro. $\mathrm{J}$ Insect Physiol. 1994; 40(6):537-547.

Fan Y, Zurek L, Dykstra MJ, Schal C. Hydrocarbon synthesis by enzymatically dissociated oenocytes of the abdominal integument of the german cockroach, Blattella germanica. Naturwissenschaften. 2003; 90:121-126. [PubMed: 12649753]

Gillespie JP, Kanost MR, Trenczek T. Biological mediators of insect immunity. Ann Rev Entomol. 1997; 42:611-643. [PubMed: 9017902]

Grzelak K, Kumaran AK. Developmental changes in the larval fat body during metamorphosis in Galleria mellonella. J Insect Physiol. 1986; 32(5):445-453.

Gutierrez E, Wiggins D, Fielding B, Gould AP. Specialized hepatocyte-like cells regulate Drosophila lipid metabolism. Nature. 2007; 445:275-280. [PubMed: 17136098]

Haunerland NH, Shirk PD. Regional and functional differentiation in the insect fat body. Ann Rev Entomol. 1995; 40:121-145.

Hetru C, Troxler L, Hoffmann JA. Drosophila melanogaster antimicrobial defense. J Infect Dis. 2003; 187(2):S327-334. [PubMed: 12792847]

Jackson A, Locke M. The formation of plasma membrane reticular systems in the oenocytes of an insect. Tissue Cell. 1989; 21:463-473. [PubMed: 18620271]

Johnson MB, Butterworth FM. Maturation and aging of adult fat body and oenocytes in Drosophila as revealed by light microscopic morphometry. J Morphol. 1985; 184:51-59. [PubMed: 3921720]

Larsen WJ. Cell remodeling in the fat body of an insect. Tissue Cell. 1976; 8(1):73-92. [PubMed: 178069]

Locke M. The ultrastructure of the oenocytes in the molt/intermolt cycle of an insect. Tissue Cell. 1969; 1:103-154. [PubMed: 18631462]

Martins GF, Pimenta PFP. Structural changes in fat body of Aedes aegytpti caused by aging and blood feeding. J Med Entomol. 2008; 45:1102-1107. [PubMed: 19058635] 
Paskewitz, SM.; Christensen, BM. Immune responses of vectors. In: Beaty, BJ.; Marquadt, WC., editors. In the biology of disease vectors. Boulder, Colorado: University Press of Colorado; 1996. p. 632

Pimenta PFP, De Souza W. Leishmania mexicana amazonensis surface charge of amastigote and promastigote formes. Exp Parasitol. 1983; 56:194-206. [PubMed: 6617803]

Raikhel AS, Lea AO. Previtellogenic development and synthesis in the fat body of a mosquito: an ultrastructural and imunocytochemical study. Tissue Cell. 1983; 15:281-300. [PubMed: 6349013]

Raikhel AS. Role of lysosomes in regulating of vitellogenin secretion in the mosquito fat body. $\mathrm{J}$ Insect Physiol. 1986a; 32(7):597-604.

Raikhel AS. Lysosomes in the cessation of vitellogenin secretion by the mosquito fat body; selective degradation of Golgi complexes and secretory granules. Tissue Cell. 1986b; 18(1):125-142. [PubMed: 3961792]

Reynolds ES. The use of lead citrate at high $\mathrm{pH}$ as an electron-opaque stain for electron microscopy. J Cell Biol. 1963; 14:208. [PubMed: 13986422]

Snigirevskaya ES, Hays AR, Raikhel AS. Secretory and internalization pathways of mosquito yolk protein precursors. Cell Tissue Res. 1997; 290:129-142. [PubMed: 9377633]

Sohal RS. Fine structural alterations with age in the fat body of the adult male housefly, Musca domestica. Z Zellforsch. 1973; 140:169-175. [PubMed: 4353958]

Stoppie P, Briers T, Huybrechts R, De Loof A. Molting hormone, juvenile hormone and the ultrastructure of the fat body of adult Sarcophaga bullata (Diptera). Cell Tissue Res. 1981; 221:233-244. [PubMed: 7307050]

Thomsen E, Thomsen M. Fine structure of the fat body of the female of Calliphora erythrocephala during the first egg-maturation cycle. Cell Tissue Res. 1974; 152 (2):193-217. [PubMed: 4434465]

Tobe SS, Davey KG. Autoradiographic study of protein synthesis in abdominal tissues of Glossina austeni. Tissue Cell. 1974; 6(2):255-268. [PubMed: 4472008]

Tucker LE. The influence of diet, age and state of hydration on $\mathrm{Na}+\mathrm{K}+$ and urate balance in the fat body of the cockroach Periplaneta americana. J Exp Biol. 1977; 71:67-79.

Tzou P, De Gregório E, Lemaitre B. How Drosophila combats microbial infection: a model to study innate immunity and host-pathogen interactions. Curr Opin Microbiol. 2002; 5:102-110. [PubMed: 11834378]

Wang Z, Haunerland NH. Ultrastructural study of storage protein granules in fat body of the corn earworm, Heliotis zea. J Insect Physiol. 1991; 37(5):353-363.

Wang Z, Haunerland NH. Fate of differentiated fat body tissues during metamorphosis of Helicoverpa zea. J Insect Physiol. 1992; 38 (3):199-213.

Wigglesworth VB. The storage of protein, fat, glycogen and uric acid in the body and other tissues of mosquito larva. J Exp Biol. 1942; 19:56-77.

Wigglesworth VB. The source of lipids and polyphenols for the insect cuticle: the role of fat body, oenocytes and oenocytoids. Tissue Cell. 1988; 20(6):919-932. [PubMed: 18620248]

Ziegler R, Ibrahin MM. Formation of lipid reserves in fat body and eggs of the yellow fever mosquito, Aedes aegypti. J Insect Physiol. 2001; 47:623-627. [PubMed: 11249951] 


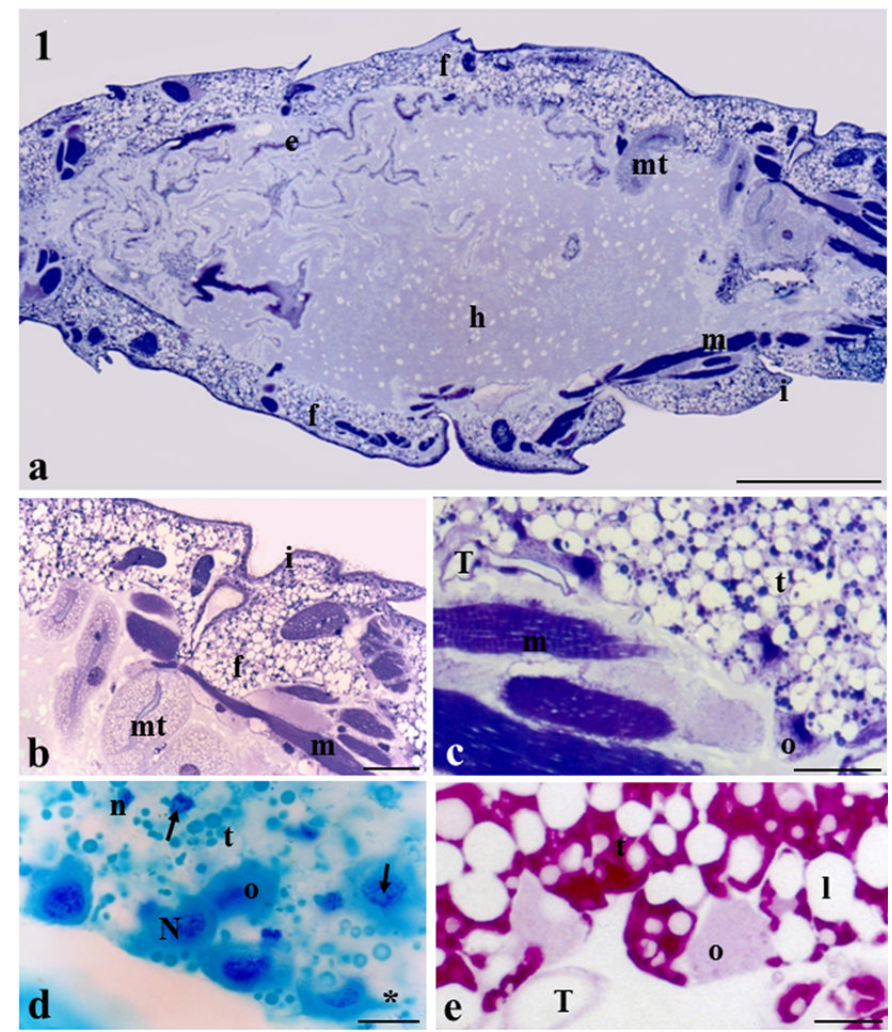

Figure 1.

Histological sections showing general and detailed views of the abdominal fat body of newly emerged A. aegypti females. Sections were stained with toluidine blue [a-c], Feulgen reaction followed by fast green [d], and PAS test [e]. [a] General view of the abdominal fat body (f) distributed beneath the integument (i). Bar=1mm; [b] Fat body (f) can be seen either in direct contact with abdominal organs, or separated by muscular layer. Bar $=200 \mu \mathrm{m}$; [c] Detailed view of the fat body with the main cell types, trophocytes ( $t$ ) and oenocytes (o). Bar $=100 \mu \mathrm{m}$; [d] Trophocytes ( $\mathrm{t}$ ) and clustered oenocytes (o) with a central nucleus $(\mathrm{N})$ and cell elongations $\left(^{*}\right)$. Note that oenocytes' nuclei $(\mathrm{N})$ are well developed in comparison to nuclei from trophocytes (n). Nucleolus within the nuclei of trophocytes and oenocytes are indicated by arrows. Bar $=30 \mu \mathrm{m}$; [e] Detailed view of PAS-positive trophocytes $(\mathrm{t})$ and the PAS-negative oenocytes (o) are shown. Bar $=30 \mu \mathrm{m}$; e-midgut epithelium; h-hemocoel; 1lipid droplet; m-muscle; mt-Malpighian tubules; T-trachea. 

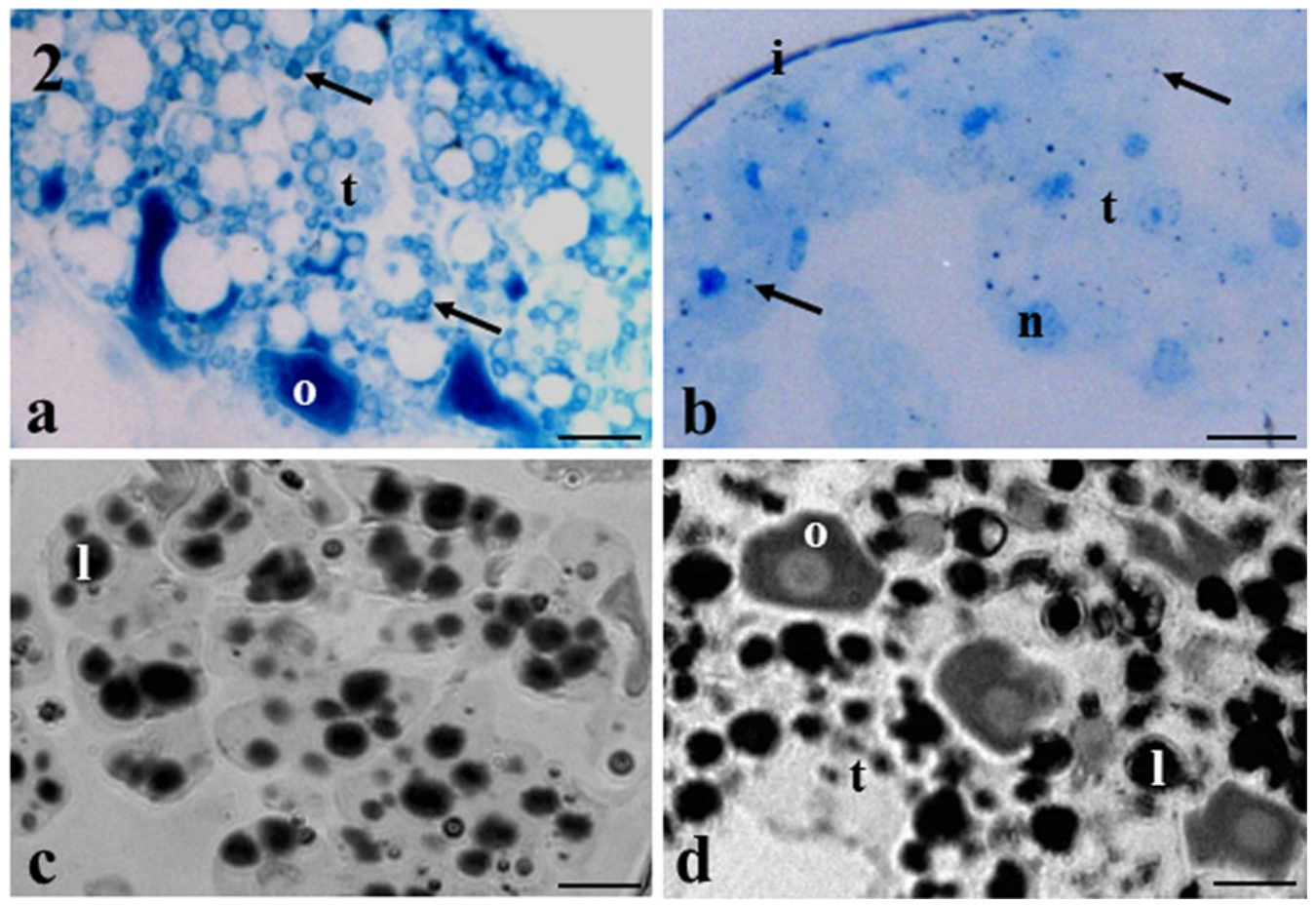

Figure 2.

Histological sections of the A. aegypti female fat bodies stained with bromophenol blue [ab] and with osmium tetroxide [c-d]. [a] Fat body of newly emerged A. aegypti female showing trophocytes $(t)$ with protein granules (arrows) with different sizes and tonalities. Oenocytes (o) are strongly uniformly stained by bromophenol blue, being difficult to distinguish their nucleus-cytoplasm limit. Bar $=40 \mu \mathrm{m}$; [b] Fat body of 18-day-old blood-fed female (four days after blood feeding) showing trophocytes (t) with few small protein granules (arrows). Bar=60 $\mu \mathrm{m}$; [c] Fat body of newly emerged female showing osmiophilic cytoplasm inclusions that correspond to lipid droplets (l) into trophocyte cytoplasm.

Bar $=40 \mu \mathrm{m}$; [d] Fat body of 18-day-old blood-fed female showing the large lipid droplets (1) into trophocytes cytoplasm (t) and the oenocytes (o) with a uniform osmiophilic cytoplasm. Bar $=20 \mu \mathrm{m}$; i-integument; n-trophocyte nucleus. 

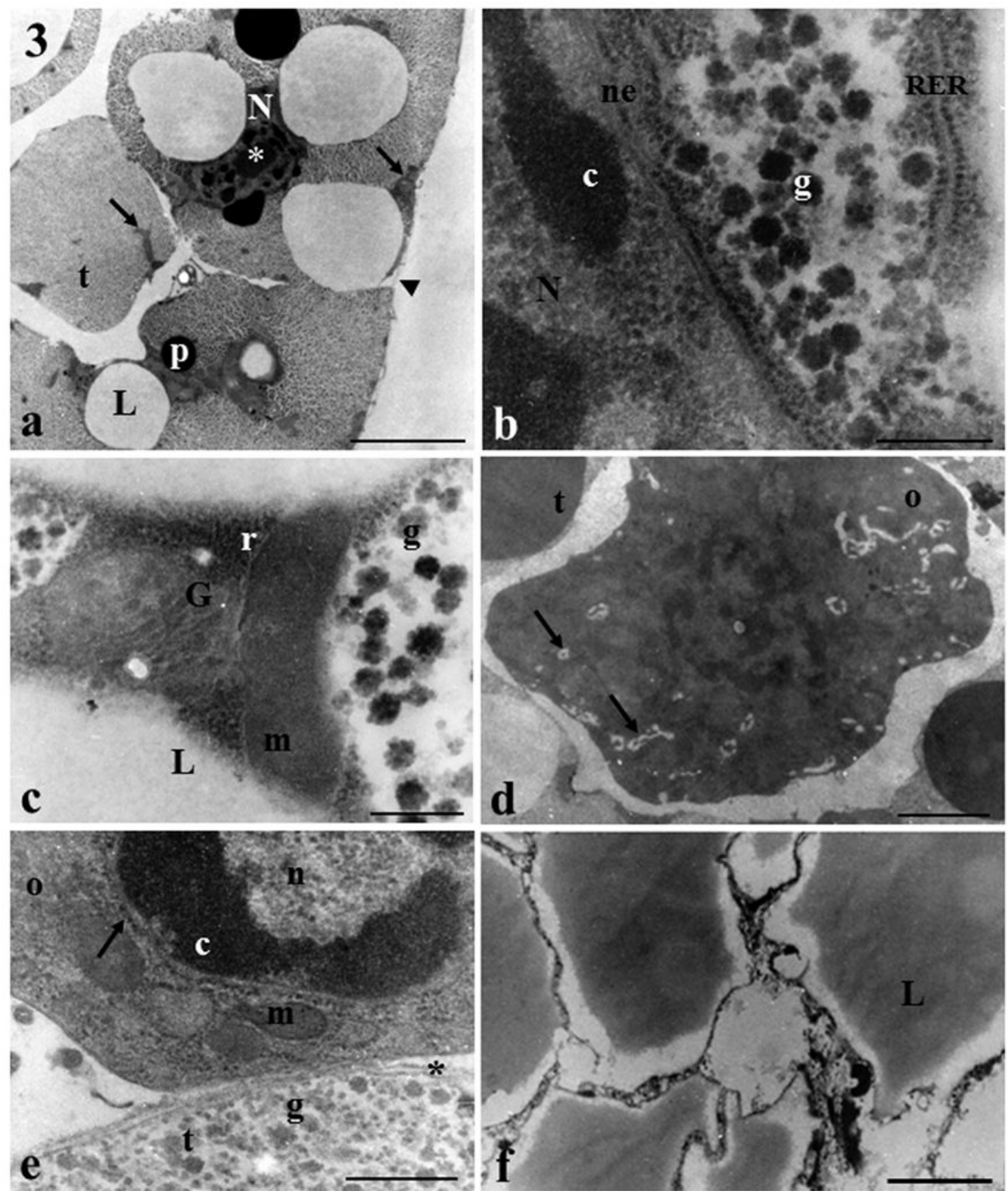

Figure 3.

TEM pictures of the fat body of A. aegypti showing details of trophocytes and oenocytes in newly emerged females [a-e] and of trophocyte in 18-day-old sugar-fed female [f]. [a] Trophocyte of newly emerged female with the cytoplasm filled with lipid droplets (L) and protein granules (p). Arrowhead indicates the external basal lamina and the arrows indicate cytoplasm regions containing ribosomes and organelles spectra. Bar $=5 \mu \mathrm{m}$; [b] Detailed view of the trophocyte cytoplasm of a newly emerged female with the rough endoplasmic reticulum (RER) and the external membrane of the nuclear envelop (ne) with the ribosomes; c-condensed chromatin inside trophocyte nucleus; g-glycogen granule; N-nucleus.

Bar=250nm; [c] Detailed view of the trophocyte cytoplasm of a newly emerged female with Golgi complex $(\mathrm{G})$ and mitochondrion $(\mathrm{m})$. Two regions can be distinguished, one filled with free ribosomes (r) and other filled with rosette-glycogen granules (g). Bar=0.3 $\mu \mathrm{m}$; [d] Oenocyte (o) of newly emerged female with the electron-dense cytoplasm and the cell membrane infoldings (arrows). Bar $=0.5 \mu \mathrm{m}$; [e] Oenocyte (o) of newly emerged female; 
Arrow - external nuclear envelope membrane with ribosomes. Bar=0.3 $\mu \mathrm{m}$; [f] Trophocyte cytoplasm of 18-day-old sugar-fed female with large lipid droplets (L) and few organelles and glycogen granules. Bar $=4 \mu \mathrm{m}$; *nucleolus; c-condensed chromatin; L-lipid droplet; $\mathrm{m}$ mitochondria; N-trophocyte nucleus; n-oenocyte nucleus; t-trophocyte. 

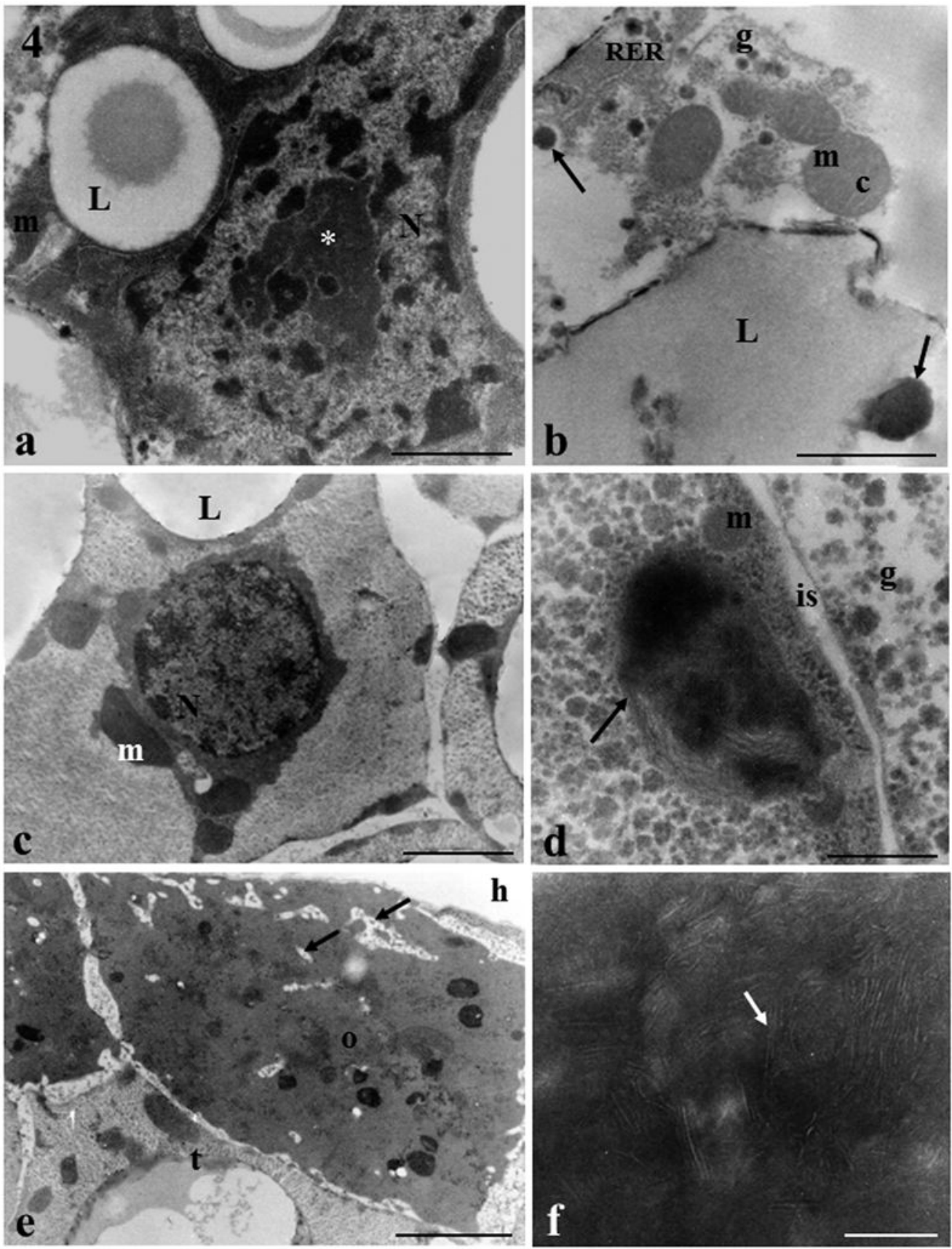

Figure 4.

TEM pictures of the fat body of 18-day-old sugar-fed [a-b] and 18-day-old blood-fed (four days after blood feeding) [c-f] A. aegypti females with details of trophocytes and oenocytes. [a] Trophocyte of 18-day-old sugar-fed female, with a polymorphic nucleus squeezed between lipid droplets (L) and a developed nucleolus $(*)$. Bar=1 $\mu \mathrm{m}$; [b] Trophocyte cytoplasm of sugar-fed female with mitochondria $(\mathrm{m})$ displaying reduced crystae and an isolated rough endoplasmic reticulum (RER). Bar $=0.5 \mu \mathrm{m}$; [c] Trophocytes of 18-day-old blood-fed female with spherical nucleus $(\mathrm{N})$, and mitochondria $(\mathrm{m})$ surrounding the nucleus or on the periphery of the cytoplasm. Bar= $2 \mu \mathrm{m}$; [d] Trophocyte of 18-day-old blood-fed female with lysosome-like structures (arrow) possibly representing organelle autophagy. 
Bar $=0.5 \mu \mathrm{m}$; [e] Oenocyte (o) of 18-day-old blood-fed female on the periphery of the fat

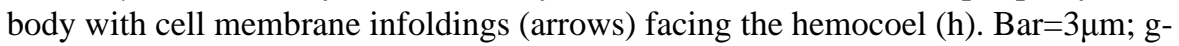
glycogen granule; i-intercellular space; L-lipid droplet; m-mitochondrion; t-trophocyte; [f] Detail of oenocyte from a 18-day-old blood-fed female showing a well-developed smooth endoplasmic reticulum (SER). Arrow indicates the SER cistern. Bar=100nm. g-glycogen; isintercellular space; L-lipid droplet; m-mitochondrion; t-trophocyte. 
Table 1

Estimated size of cell nucleus in A. aegypti trophocytes and oenocytes.

\begin{tabular}{lllc}
\hline cell type & \multicolumn{3}{l}{ A. aegypti females } \\
\hline & newly emerged $(\mathrm{n}=6)$ & \multicolumn{2}{c}{ 18-day-old $(\mathbf{n}=6)$} \\
\cline { 2 - 4 } & & sugar-fed & blood-fed \\
\cline { 2 - 4 } trophocytes & $13.55 \pm 2.23(\mathrm{~N}=241)$ & $17.06 \pm 2.93(\mathrm{~N}=249)$ & $15.65 \pm 2.49(\mathrm{~N}=229)$ \\
oenocytes & $20.42 \pm 2.53 *(\mathrm{~N}=40)$ & $19.16 \pm 3.12 *(\mathrm{~N}=30)$ & $14.5 \pm 2.17(\mathrm{~N}=22)$ \\
\hline
\end{tabular}

Size was estimated based on the diameter of the nucleus from each cell (indicated in $\mu \mathrm{m}$, mean \pm SD). Shown are: $\mathrm{N}$, the number of cell nuclei analyzed; and n, the number of A. aegypti females from which trophocytes and oenocytes were dissected. The mean diameter for each group also was determined.

indicate the calculated mean is not statistically different (Tukey test $\mathrm{p}>0.05$ ). Mean values are statistically different for all other samples (Tukey test $\mathrm{p}<0.05)$. 